\title{
Tecnologías de la información y la comunicación como herramienta para enseñar matemáticas ${ }^{1}$
}

\section{Information and communication technologies as a tool to teach mathematics}

DOI: http://dx.doi.org/10.17981/cultedusoc.9.3.2018.86

Artículo de investigación. Fecha de recepción: 15/06/2018. Fecha de aceptación: 27/11/2018

\section{Madelis González-Escorcia ${ }^{2}$;}

Nohora Jiménez-Mercado y Adriana Rangel-Fontalvo ${ }^{3}$

Institución Educativa Departamental Juan Manuel Rudas sede La Milagrosa (Colombia) madelisp@hotmail.com

Para citar este artículo:

González-Escorcia, M., Jiménez-Mercado, N y Rangel-Fontalvo, A. (2018). Tecnologías de la información y la comunicación como herramienta para enseñar matemáticas. Cultura. Educación y Sociedad 9(3), 733-740. DOI: http://dx.doi.org/10.17981/cultedusoc.9.3.2018.86

\section{Resumen}

Las matemáticas son un área fundamental en el aprendizaje del estudiante, fomentando el desarrollo de procesos cognitivo, como el pensamiento lógico y el razonamiento ordenado. Por lo tanto la presente investigación tuvo como objetivo implementar las tecnologías de la información y la comunicación TIC como herramienta para la enseñanza de las matemáticas. La investigación es de tipo cualitativa, apoyada en los lineamientos de la IEP, de alcance descriptivo. La unidad de análisis estuvo conformada por (40) estudiantes de tercero y cuarto de primaria de la Institución Educativa Juan Manuel Rudas, sede La Milagrosa. Los resultados dejaron en evidencia que los mejoraron su rendimiento académico con las estrategias pedagógicas apoyadas en las TIC que los maestros pusieron en práctica, no obstante se debe dar continuidad a las nuevas alternativas de enseñanza, de acuerdo al contexto y a las necesidades académicas.

Palabras clave: tecnologías de la información y la comunicación, herramientas pedagógicas, matemáticas.

\section{Abstract}

Mathematics is a fundamental area in student learning, fostering the development of cognitive processes, such as logical thinking and ordered reasoning. Therefore, the present investigation had the objective of implementing ICT information and communication technologies as a tool for the teaching of mathematics. The research is of a qualitative nature, supported by the IEP guidelines, which are descriptive in scope. The analysis unit consisted of (40) third and fourth grade students of the Juan Manuel Rudas Educational Institution, La Milagrosa. The results showed that they improved their academic performance with the pedagogical strategies supported by the ICTs that the teachers put into practice, nevertheless they must give continuity to the new teaching alternatives, according to the context and the academic needs.

Keywords: information and communication technologies, pedagogical tools, mathematics.

1 Este artículo ha sido derivado del Programa de Fortalecimiento de la Cultura Ciudadana y Democrática CT+I a través de la IEP apoyada en TIC en el Departamento de Magdalena: CICLON

2 Líder del grupo de investigación "Los Matemoustros".

3 Docentes de la Institución Educativa Departamental Juan Manuel Rudas sede La Milagrosa y miembros del Grupo “Los Matemoustros”.

- The author; licensee Universidad de la Costa - CUC.

Cultura, Educación y Sociedad vol. 9 no. 3, pp. 733-740. Diciembre, 2018

Barranquilla. ISSN 2389-7724 Online 


\section{Introducción}

El presente estudio tuvo como objetivo implementar las tecnologías de la información y la comunicación TIC como herramienta para la enseñanza de las matemáticas. El intereses del estudio subyace de la realidad que actualmente presenta la Institución Educativa Juan Manuel Rudas, sede La Milagrosa, en donde inicialmente se observó un bajo rendimiento académico y puntajes inferiores en las pruebas saber, para el área de matemáticas en los estudiantes, motivo por el cual se buscó promover estrategias pedagógicas innovadoras para fortalecer las competencias en esta asignatura, entendiendo que el desempeño de los estudiantes en un área depende de factores personales como el interés y la motivación, sin embargo estos se pueden promover por medio del uso de las TIC, debido a que la globalización ha traído la inclusión de las nuevas tecnologías como herramientas de apoyo y los estudiantes en la actualidad son considerados nativos digitales.

Actualmente la sociedad del conocimiento ha transformado el mundo educativo, donde la ciencia, la tecnología y la innovación inciden en diferentes de forma significativa en la vida de los estudiantes, hecho que ha producido un cambio en los pensamientos y acciones de los individuos frente al proceso de enseñanza (Cárdenas y Angulo, 2016)

Según Ramírez y Ampudia, (2018). La tecnología, es una herramienta que promueve y aumenta las oportunidades de los países en vías de desarrollo a través del conocimiento. En las instituciones educativas estas han tomado fuerza, puesto que la combinación de información, contexto y experiencia, permiten alcanzar los objetivos de las diferentes áreas de estudio, como medio para obtener y administrar el conocimiento requerido en los estudiantes.
Por otro lado, Avendaño, Cortes y Guerrero (2015), expresan que el uso de las tecnologías de la información y la comunicación (TIC) tienen un papel importante en el desarrollo de competencias educativas y sociales, como factores predictores del desempeño académico en estudiantes de básica primaria.

En la actualidad el manejo de la tecnología de una forma eficaz, es una habilidad necesaria para desenvolverse en la sociedad, este cambio de paradigma social trae consigo el cambio en el paradigma educativo. Por lo cual, la educación realizar inversiones económicas y políticas, donde su papel se fundamente en la capacidad de producir sus propias herramientas tecnológicas. Y la actualización del cuerpo docente para que se encuentre en la capacidad de responder al desafío de la actualización tecnológica (Riveros y Mendoza, 2004).

Por lo anterior, es importante realizar uso correcto de las TIC que se implemente como una herramienta de interacción entre docentes y estudiantes. Enfocándose en estos últimos como la parte más importante del proceso de aprendizaje, donde el docente debe asumir un rol de apoyo, facilitando el acceso a la información. Utilizar las TIC en el aula de clase permite generar espacios de aprendizaje colaborativo (Pichardo y Puente, 2012).

Actualmente, el aprendizaje de las matemáticas, puede convertirse en un escenario de aprendizaje participativo y de interacción con el apoyo de las TIC, a travez de videos, canciones, juegos, búsquedas en tempo real etc. Logrando remplazar las actividades magistrales, y motivando al estudiante a la constante participación.

La educación como la sociedad está cambiando continuamente, la tecnología ha creado nuevas exigencias sociales y laborales, por lo que al mismo tiempo se exigen cosas nuevas en la educación para dar atención a estas necesidades. De la 
realización de esta investigación se puede concluir entre otras cosas, que para la implementación de las TIC hay diferentes medios disponibles, desde softwares especializados hasta video juegos y redes sociales. Lo cual se sustenta en los planteamientos de Cárdenas y Angulo (2016), quienes plantean que en la sociedad del conocimiento ha transformado el mundo educativo, donde la ciencia, la tecnología y la innovación inciden en diferentes de forma significativa en la vida de los estudiantes, hecho que ha producido un cambio en los pensamientos y acciones de los individuos frente al proceso de enseñanza.

Además, las TIC son una herramienta que tiene el profesor para llevar a sus estudiantes conceptos que muy abstractos y difíciles de comprender con una imagen práctica y contextualizada. La implementación de las TIC ayudó no solo a incluir tecnología en las clases, sino a generar interés en los alumnos y plantear un modelo educativo totalmente diferente, donde el principal actor es el alumno y no el profesor. Al poner en práctica el proyecto se encontró que un obstáculo importante es la falta de recursos y muchas veces de capacitaciones adecuadas a los maestros, por lo que son puntos que fortalecer previo a comenzar un proyecto de implementación de las TIC en cualquier institución. Lo anterior confluye con lo mencionado por Pichardo y Puente (2012), quienes plantean la importancia de realizar uso correcto de las TIC que se implemente como una herramienta de interacción entre docentes y estudiantes. Enfocándose en estos últimos como la parte más importante del proceso de aprendizaje, donde el docente debe asumir un rol de apoyo, facilitando el acceso a la información. Concluyendo en la idea que utilizar las TIC en el aula de clase permite generar espacios de aprendizaje colaborativo, permitiendo el intercambio de saber y conocimiento.
Matemática educativa: modelos pedagógicos basados en tecnología para la enseñanza de Matemáticas

El constructivismo es; una de las posturas epistemológicas que se encuentra inmersa la enseñanza de la matemática, con el fin de lograr que el proceso de aprendizaje se logre en todos los ámbitos de intervención (Kilpatrick, Gómez y Rico, 1995) Lo anterior va en concordancia con el propósito de la investigación como estrategia pedagógica en donde el eje fundamental es la enseñanza a través de la experiencia en todos los contextos educativos.

Los supuestos de la teoría en mención implican que:

- El Ser Humano posee estructuras cognitivas, necesarias para los procesos de aprendizaje de las matemáticas; las cuales están en permanente desarrollo.

- El conocimiento matemático se construye mediante abstracciones reflexivas

- La tecnología es una herramienta importante en la actualidad para el desarrollo de competencias en las matemáticas.

\section{Geogebra para el aprendizaje \\ de la enseñanza}

Geogebra es un software matemático interactivo libre utilizado en colegios y universidades es programado en Java y escrito en Java y HTML5. Está disponible en cerca de 50 idiomas y ha ganado premios como el Microsoft Education Hohenwarter. Ha resultado ser un recurso abierto y de gran utilidad, logrando manejar diversos niveles en actividades de aprendizaje Es un recurso muy importante que se tiene disponible para su uso, siendo un programa bastante intuitivo y en el que se pueden manejar niveles en las actividades de aprendizaje (Ávila y Moreno, 2015) 
Según el estudio realizado por García (2011) el software tiene fortalezas a la hora de ser utilizado en el aula de clase, a continuación, se muestran los resultados más significativos de su estudio:

"Los análisis más detallados, realizados para la muestra de estudiantes y estudio de casos, revelaron la existencia de otro atributo de Geogebra que también contribuyó a la mejora de este componente y que la lectura de los diarios grupales confirma: la interactividad o retroalimentación inmediata y efectiva que permite la toma de conciencia $y$ conciliación de los errores cometidos" (p. 225).

Además, en su estudio revela que, para muchos de los estudiantes, la retroalimentación recibida por el programa cuando experimentaban o testaban alguna hipótesis, hizo posible que vencieran sentimientos de incapacidad ante la realización de algunas tareas. De igual manera, con el uso del software fue posible mejorar las dificultades y lograr que los estudiantes ganaran cierta confianza ante la resolución de problemas de manera individual (García, 2011)

\section{Utilidad de lo aprendido para el futuro del alumno}

Es importante que el alumno obtenga las habilidades en el aula de clase, que seguramente le servirán en su trabajo o educación continuada. Según Riveros y Mendoza (2005).

"En el marco de esta sociedad del conocimiento, los alumnos deben aprender con las herramientas que seguramente se encontraran más tarde en sus puestos de trabajo. Por tanto, están llamados a utilizar la tecnología como un medio y no como un fin, de tal forma que los computadores, las redes como la Internet, multimedias, hipermedias, la realidad virtual y otros, sean medios con los cuales puedan aprender y pensar" (p. 25).
Entendiendo que el profesor no podrá enseñar específicamente a cada alumno aquellos conocimientos que le servirán en un futuro, el objetivo debe ser lograr que el alumno aprenda a aprender, buscando que desarrollen diferentes habilidades fácilmente. Contando con esta habilidad, el alumno podrá implementar sus herramientas en cualquier campo que se quiera desempeñar (Riveros y Mendoza, 2005).

\section{Requerimientos para una integración completa de las TIC en las escuelas}

La implementación de las TIC de una manera amplia requiere de especificaciones que permitan la planificación y organización previa de los contenidos.

Algunos de los requerimientos planteados en el proyecto según Morrissey, (2008). serían los siguientes:

- La disponibilidad y provisión de adecuados, confiables y suficientes recursos TIC, tanto para estudiantes como para docentes.

- Inclusión de las TIC en los planes de estudio en las escuelas y la garantía de ser empleadas en espacios de enseñanza-aprendizaje.

- Uso de las TIC en procesos de evaluación de aprendizajes.

- Apoyo de las TIC en desarrollo profesional de los docentes de la institución.

Sin embargo, es fundamental que el gobierno colombiano establezca algunas pautas básicas y el consejo nacional de profesores puede incluir a las TIC como eje del aprendizaje en las clases de matemáticas. Un ejemplo de esto se ve en Estados Unidos, con el Consejo Estadounidense de Profesores de Matemática (NCTM) el cual establece, una serie de pautas relativas a la educación matemática en la actualidad. Encontrando que la tecnología es necesaria para mejorar la enseñanza de las matemáticas puesto que el componente 
innovador de las TIC genera un impacto positivo en el aprendizaje de los estudiantes, sustentada desde el paradigma constructivista (Téliz, 2015).

Además, es importante para el sector educativo público que las escuelas de básica primaria y secundaria analicen los logros obtenidos en las instituciones y traten de adaptar dentro de lo posible medidas de acuerdo al éxito de estas en el ámbito académico, y la necesidad del alumno de estar familiarizado con estas desde la escuela para su desempeño en la educación universitaria. Por lo cual es necesario entender la manera de aplicar los nuevos conceptos de la educación teniendo en cuenta la experiencia previa de otros entes educativos (Camacho, 2005).

\section{La computadora como}

herramienta tecno-pedagógica

Un claro obstáculo al momento de la implementación de las TIC es la falta de recursos, en los países latinoamericanos entre ellos Colombia, se ve reflejada esta problemática en muchas escuelas de básica primaria y secundaria, principalmente en las entidades públicas de educación no cuentan con las condiciones tecnológicas para el funcionamiento a cabalidad de los copmtutadores, tabletas etc. Lo anterior va en concordancia con estudio realizado por Román y Murillo (2014), cuyos resultados indican que el $28 \%$ de las escuelas de latinoamérica no dispone de computadoras con acceso a Internet; el 23,2 \% no dispone de ninguna computadora para uso de los estudiantes y un 23,5 \% tampoco cuenta con computadoras con acceso a Internet para los docentes.

Por otro lado, la actitud de los profesores frente a las TIC es un factor importante que tratar, ya que sin ellos no se podría llevar a cabo esta metodología educativa. Dentro del contexto global los profesores tienen actitudes positivas frente al uso de las TIC, pero se presentan diferentes tipos de obstáculos que entorpecen su labor al querer hacer uso de la nueva metodología. (Lucena, Martín y Díaz, 2002).

Una posible solución a esto es el uso del aprendizaje web, a través de videos y pruebas virtuales donde los entornos de aprendizaje virtual, en contenido multimedia basados en videoclips de clases de matemáticas diseñadas para desarrollar competencias profesionales de los futuros maestros, permiten crear estrategias de Enseñanza-Aprendizaje que promueven la generación de nuevas prácticas que capacitan maestros para aprender a utilizar las herramientas tecnológicas específicas para el profesor de matemáticas (Valls, Llinares y Callejo, 2007).

Por su parte, el uso de redes sociales educativas sirve como complemento a las TIC en el proceso de enseñanza puesto que estas permiten la interacción entre docentes y estudiantes posibilitando la comunicación, que se ve incrementada por la creación de grupos de trabajo, pero a su vez estas tienen un efecto de atracción social para los alumnos que implica un acercamiento del aprendizaje contextual con el teórico (Haro, 2009).

\section{El uso de los video juegos en las escuelas}

Una forma de implementar las TIC, especialmente a los alumnos de menor edad es a través de aquello que más los atrae, por ello se justifica el uso de video juegos con el objetivo de enseñar aquel contenido requerido para la clase. Según Gros \& Garrido, (2008). Los videojuegos tambié herramientas tecnológicas que se emplean frecuentemente para el desarrollo de contenidos en las aulas de clase. Su uso no necesaria y exclusivamente responde a contenidos curriculares concretos, sino que también aporta en el desarrollo de competencias tecnológicas en sus múltiples aplicaciones y actividades. 
Cabe resaltar que es indispensable hacer una exhaustiva selección de los videosjuegos apropiados para los niños, teniendo en cuenta su edad, grado escolar y las competencias que se desean desarrollar, con el fin de que el profesor pueda aprovechar el espacio y herramientas, y con ello generar espacios en los que el estudiante pueda enfrentarse a un sistema interactivo, multidimensional y multimedia, en toda su complejidad.

Actualmente la tecnología no nos limita tanto a la hora de realizar un videojuego, presentando una muy amplia gama de posibilidades para su implementación en campos como la educación. Teniendo en cuenta el acceso a la tecnología de los estudiantes, se debe preguntar si se cuentan con los medios para la realización de video juegos educativos que atraigan a la población objetivo y sean lo suficientemente complejos para transmitir de la mejor forma el conocimiento que se busca entregar a través de ellos.

Por su parte Gros (2004) afirma que los entornos multimedia resultan ser unas herramientas factibles para el apoyo en la puesta en marcha de nuevos modelos cognitivos. Los videojuegos pasan a ser complejas producciones semejantes a películas de grandes productoras. Con lo dicho anteriormente se observa que la tecnología más que un limitante es una herramienta de múltiples posibilidades que entrega a los docentes un sin número de posibilidades de llevar el conocimiento a los estudiantes.

\section{Metodología}

La investigación es abordada desde una mirada cualitativa, de alcance descriptico, guiada bajo los lineamientos metodológicos de la Investigación como Estrategia Pedagógica IEP, tomando la indagación como estrategia que permite integrar la teoría y los aprendizajes cotidianos de la experiencia en grupos infantiles y juveniles, promulgando el desarrollo de saber y conocimiento científico (Mejía y Manjarrés, 2010).

\section{Participantes}

La unidad de análisis estuvo conformada por cuarenta (40) estudiantes de tercero y cuarto de primaria de la Institución Educativa Juan Manuel Rudas, sede La Milagrosa.

\section{Técnicas e instrumentos}

Para la recolección de la información se utilizaron técnicas observación participante, entrevistas, registradas en bitácoras, cuaderno de notas y guía de preguntas de las experiencias observadas en el proceso de implementación de las tecnologías de la información y la comunicación como herramienta para enseñar matemáticas.

\section{Resultados}

En cuanto a la información sociodemográfica la población se encuentra ubicada en Remolino un pueblo ribereño, bañado por el Rio Magdalena, ubicado al noroccidente del departamento del Magdalena. Las familias de esta comunidad son de escasos recursos económicos, la mayoría solo laboran en épocas de cosecha, otros se trasladan a trabajar al Departamento del Atlántico, dejando a sus hijos a cargo de familiares.

De acuerdo a los resultados de las pruebas saber y el rendimiento académico, se iniciaron las observaciones y registros desde las metodologías impartidas por los docentes y el aprendizaje de los estudiantes en el área de las matemáticas. Con este diagnóstico, los docentes realizaron una revisión sistemática de diferentes programas basados en la inclusión de las TIC al aula de clase para la enseñanza de matemáticas, se tomaron acciones propuestas por la IEP donde mediante el uso de las tablets los estudiantes indagaron sobre la 
temática abordada según los contenidos curriculares y su aplicación a la vida cotidiana, seguidamente se buscaron juegos interactivos que favorecieran el desarrollo de dichas competencias, donde a través del proceso de observación se identificó que el proceso de enseñanza basado en una metodología teórico-práctica, permite a los estudiantes convertirse en sujetos activos en la construcción de su aprendizaje a partir de procesos de indagación, observación y análisis, lo que ayudó a crear criterios propios sobre situaciones particulares del contexto.

Partiendo de estas estrategias basadas en la integración de las TIC al aula, se logró analizar y utilizar las matemáticas de forma práctica, contextualizada y divertida, contrario a la concepción que tenían los estudiantes sobre el aprendizaje de esta área, de esta forma los estudiantes de tercero y cuarto grado de primaria de IED Juan Manuel Rudas sede La Milagrosa, lograron aprender componentes básicos de la asignatura mediante las estrategias didácticas utilizadas en el aprendizaje de la misma, donde los docentes se encuentran en un proceso de capacitación permanente por medio de herramientas virtuales que les permiten diseñar actividades para desarrollar habilidades de análisis y comprensión en situaciones matemáticas, apropiadas para los contenidos curriculares que se estén trabajando según el grado escolar.

\section{Discusión}

Las TIC como herramientas pedagógicas han resultado ser una de las salidas para el aumento en cuanto al rendimiento académico y por ende la generación de novedosos aprendizajes. Los resultados permiten ver que efectivamente, los estudiantes tenían bajos niveles de motivación frente al área de matemáticas, lo que se observaba las pruebas estandarizadas.
Con la inclusión de las TIC por parte del profesorado en las estrategias de enseñanza, se pudo evidenciar mayor participación y motivación por parte de los estudiantes, sin embargo se debe dar continuidad a nuevas estrategias no perdiendo por ningún motivo la mirada pedagógica de las tecnologías de la información y la comunicación.

El éxito de la enseñanza dependerá del esfuerzo mancomunado de la comunidad educativa, así mismo de la adecuación de escenarios educativos propicios para la promoción nuevos saberes, por ejemplo teniendo los insumos tecnológicos, y el acceso a ellos para llevar a la práctica nuevas maneras de aprender.

\section{Referencias}

Avendaño, I., Cortés, O. y Guerrero, H. (2015). Competencias sociales y tecnologías de la información y la comunicación como factores asociados al desempeño en estudiantes de básica primaria con experiencia de desplazamiento forzado. Diversitas, 11(1), 13-36.

Ávila, M. y Moreno, A. (2015). Implementación del software libre Geogebra como herramienta en el aula para la enseñanza de las matemáticas en educación básica secundaria en la ciudad de Fusagasugá. Revista Colombiana de Matemática Educativa. 1(1). 664-668.

Camacho, M. (2005). Enseñanza y aprendizaje del análisis matemático haciendo uso del CAS. En, Actas del IX Simposio de la SEIEM. (97-110). Córdoba.

Cárdenas, N. y Angulo, F. (2016). Análisis de las dimensiones de adaptación, mejoramiento e innovación en los procesos de aprendizaje tecnológico. Cultura Educación y Sociedad, 7(2), 139-149.

García, M. (2011). Evolución de actitudes y competencias matemáticas en estudiantes de secundaria al introducir Geogebra en el aula. [Doctoral dissertation], Universidad de Almería, Almeria. 
Gros, B. y Garrido, J. (2008). "Con el dedo en la pantalla": el uso de un videojuego de estrategia en la mediación de aprendizajes curriculares. Teoría de la Educación. Educación y Cultura en la Sociedad de la Información, 9(3). 108129.

Gros, B. (2004). Pantallas, juegos y educación. La alfabetización digital en la escuela. Bilbao: Desclée.

Haro, J. (2009). Las redes sociales aplicadas a la práctica docente. Didáctica, innovación y multimedia, (13). 1-8.

Lucena, F., Martín, F. y Díaz, I. (2002). Las actitudes de los docentes hacia la formación en tecnologías de la información y comunicación (TIC) aplicadas a la educación. Contextos educativos. Revista de educación, (5), 253-270.

Mejía, M., y Manjarrés, M. (2010). La Investigación como Estrategia Pedagógica. Bogotá, D.C.: Programa Ondas - Colciencias.

Morrissey, J. (2008). El uso de TIC en la enseñanza y el aprendizaje: cuestiones y desafíos. En, Unicef, Seminario internacional. Cómo las TIC transforman las escuelas. (81-90). Buenos Aires, Argentina.

Pichardo, I. y Puente, Á. (2012). Innovación Educativa: Uso de las TIC en la enseñanza de la Matemática Básica. Edmetic, $1(2), 127-144$.
Ramírez, R. y Ampudia, D. (2018). Factores de Competitividad Empresarial en el Sector Comercial. Revista Electrónica de Ciencia y Tecnología del Instituto Universitario de Tecnología de Maracaibo (RECITIUTM), 4(1). 16-32.

Riveros, V. y Mendoza, M. (2005). Bases teóricas para el uso de las TIC en Educación. Encuentro educacional, 12(3).

Rojano, T. (2003). Incorporación de entornos tecnológicos de aprendizaje a la cultura escolar: proyecto de innovación educativa en matemáticas y ciencias en escuelas secundarias públicas de México. Revista iberoamericana de Educación, 33(3), 135-165.

Román, M. y Murillo, F. (2014). Disponibilidad y uso de TIC en escuelas latinoamericanas: incidencia en el rendimiento escolar. Educação e Pesquisa, 40(4).

Téliz, F. (2015). Uso didáctico de las TIC en las buenas prácticas de enseñanza de las matemáticas: Estudio de las opiniones y concepciones de docentes de educación secundaria en el departamento de Artigas. Cuadernos de Investigación Educativa, 6(2), 13-31.

Valls, J., Llinares, S. y Callejo, M. (2006). El uso de videoclips para una práctica reflexiva. En, JAEM, XIII Jornadas de Aprendizaje y Enseñanza de las Matemáticas. (25-48). Granada: Grupo Proyecto Sur. Recuperado de https:// core.ac.uk/download/pdf/16355010.pdf 